\title{
Analysis of Three-Dimensional Spatial Selectivity for Rician Channel
}

\author{
Derong DU, Xiaoping ZENG, Xin JIAN, Feng YU, Lijuan MIAO \\ College of Communication Engineering, Chongqing University, Chongqing 400044, China \\ $\left\{d r \_d u, z x p, j i a n x i n, y u f c h n\right.$, miaolijuan $\} @$ cqu.edu.cn \\ Submitted May 12, 2017 / Accepted November 8, 2017
}

\begin{abstract}
The spatial selectivity of multipath fading determines the spatial diversity strategy to increase the performance of the communication system. This paper analyses the three-dimensional (3-D) spatial selectivity of Rician channel to strive to alleviate the current lack of analytical studies. A 3-D multipath angular power density (APD) model for Rician channel is proposed. Analytical expressions of the 3-D multipath shape factors are given based on the APD using the multipath shape factors theory. Finally, some important spatial fading statistics like the fading rate variance, level crossing rate ( $L C R)$, average fade duration ( $A F D)$, spatial correlation and coherence distance are derived, and the analysis on the impact of 3-D spatial angular directions on these spatial fading statistics is presented through simulations. The results would provide useful insight on quantifying and simplifying the analysis and design of the 3-D multiple input multiple output (MIMO) beamforming technology and smart antenna arrays.
\end{abstract}

\section{Keywords}

Three-dimensional spatial selectivity, Rician channel, multipath shape factors, angular power density

\section{Introduction}

One of the key characteristics of a mobile communication channel is multipath fading due to the reflection, diffraction and scattering phenomena of radio waves. The spatial selectivity of multipath fading impacts on the performance of the spatial diversity system. Therefore, a general characterization of the spatial selectivity is needed. In [1], the multipath shape factors theory was proposed, and the factors of angular spread, angular constriction and azimuthal direction of maximum fading were shown to describe the statistics of received signal fluctuations in a two-dimensional (2-D) channel. The theory can quantify and greatly simplify the description of multipath fading statistics of non-omnidirectional channels, so many researchers have analyzed the spatial selectivity of multipath fading based on the theory. Applications of these shape factors in Rician and Nakagami-m channels were discussed in [2] and [3]. Power angle profiles were measured in different indoor locations, and detailed angle-of-arrival (AoA) shape factors describing the spatial properties of the millimeter wave channel at $60 \mathrm{GHz}$ were derived in [4] and [5]. Quantitative analysis of some notable scattering models and various spatial channel measurements were presented using multipath shape factors in [6] and [7]. The shape factor of angle spread was evaluated to compare the different directional multipath characteristics in the urban and suburban scenarios in [8]. All previously reported works in [1-8] considered 2-D multipath channels. However, in urban measurements, typically $90 \%$ of the received power is contained in paths with elevations up to $40^{0}$ [9]. Therefore, the 2-D multipath channel assumption is acceptable for some suburban areas but not acceptable for densely populated built-up urban regions, and this gives a motivation for the analysis of multipath channels in the three-dimensional (3-D) space. An alternative definition in spherical coordinates of the angular spread developed in [1] was given in [10], and a similar approach in Cartesian coordinates was followed in [11] for quantification of the 3-D directionality of the multipath power and variability of the second-order fading statistics. In [12], a 3-D multipath shape factors theory was developed based on the work in [1], and analytical expressions for the level crossing rate (LCR), average fade duration (AFD) and approximate envelope correlation were derived for a Rayleigh channel. For a 3-D air-to-ground channel model, the impact of physical channel's parameters on the multipath shape factors defined in [1] was analyzed for azimuth and elevation planes separately in [13].

Owing to flexibility and suitability, the Rician fading is often used to model the wireless propagation comprising a line-of-sight (LoS) component and a scattered non-LoS (NLoS) component [14-16]. However, as mentioned above, few works have analyzed the 3-D spatial selectivity of Rician channel. This paper strives to alleviate the current lack of analytical studies, and the contributions and novelties of this paper are summarized as follows.

- A 3-D multipath angular power density (APD) model is proposed for the spatial selectivity analysis of Rician channel; 
- The 3-D multipath shape factors are derived based on the proposed APD and greatly simplify the descriptions of some important spatial fading statistics;

- The analysis results would provide useful insight on the design of 3-D multiple-input-multiple-output (MIMO) beamforming technology and smart antenna array [17-19] for fifth generation (5G) networks.

The remainder of the paper is organized as follows. Section 2 presents the 3-D multipath APD model and the corresponding multipath shape factors of the Rician channel. Section 3 derives some important spatial fading statistics like the fading rate variance, LCR, AFD, spatial correlation and coherence distance. Discussions on obtained simulation results are provided in Sec. 4. Finally, conclusions are mentioned in Sec. 5.

\section{Three-Dimensional Multipath An- gular Power Density and Multipath Shape Factors}

A Rician channel model results from the addition of a single LoS wave and numerous diffusely scattered NLoS waves. It is assumed that the AoA of $\operatorname{LoS}$ wave is $\left(\theta_{0}, \phi_{0}\right)$, and the AoA power of NLoS waves is evenly distributed in 3-D sphere space as shown in Fig. 1.

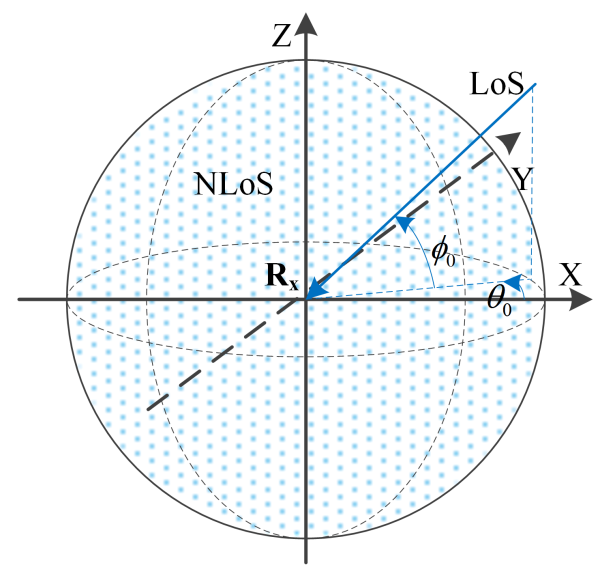

Fig. 1. The AoA of 3-D Rician channel.

Then the Rician channel can be modeled by the following 3-D multipath APD comprising a LoS component and a NLoS component [1]:

$$
\begin{aligned}
p(\theta, \phi) & =P_{\mathrm{NLoS}} f_{\mathrm{NLoS}}(\theta, \phi)+P_{\mathrm{LoS}} f_{\mathrm{LoS}}(\theta, \phi) \\
& =\frac{P_{\mathrm{T}}}{K+1} \times \frac{\cos \phi}{4 \pi}+\frac{P_{\mathrm{T}} K \delta\left(\theta-\theta_{0}, \phi-\phi_{0}\right)}{K+1} \\
& =\frac{P_{\mathrm{T}}}{4 \pi(K+1)}\left[\cos \phi+4 \pi K \delta\left(\theta-\theta_{0}, \phi-\phi_{0}\right)\right](1)
\end{aligned}
$$

where $P_{\mathrm{T}}$ is the total power in a local volume, $K$ is the Rician $\mathrm{K}$-factor, $P_{\mathrm{NLoS}}=P_{\mathrm{T}} /(K+1)$ is the power of NLoS waves, $P_{\mathrm{LoS}}=P_{\mathrm{T}} K /(K+1)$ is the power of $\operatorname{LoS}$ wave, $\theta \in[0,2 \pi]$ is the azimuth angle, $\phi \in[-\pi / 2, \pi / 2]$ is the elevation angle,
$f_{\mathrm{NLOS}}(\theta, \phi)=\cos \phi / 4 \pi$ is the APD of NLoS waves [20], and $\delta($,$) is the 2-D Dirac delta function which is used to describe$ the APD $f_{L o S}(\theta, \phi)$ of LoS wave.

Therefore, according to the definition in [12], the six 3-D multipath shape factors can be calculated as follows based on the APD in (1) to quantify and simplify the spatial fading statistics of the Rician channel.

Angular spread:

$$
\Lambda=\sqrt{1-\frac{\left(S_{1}^{0}\right)^{2}+\left|S_{1}^{1}\right|^{2}}{\left(S_{0}^{0}\right)^{2}}}=\frac{\sqrt{\pi\left(\pi+8 K \cos \phi_{0}\right)}}{\pi+4 K \cos \phi_{0}},
$$

Elevational constriction:

$$
\begin{aligned}
\xi & =\frac{\frac{3}{2} S_{2}^{0} S_{0}^{0}-\left(S_{1}^{0}\right)^{2}+\frac{1}{2}\left|S_{1}^{1}\right|^{2}}{\left(S_{0}^{0}\right)^{2}-\left(S_{1}^{0}\right)^{2}-\left|S_{1}^{1}\right|^{2}} \\
& =\frac{\pi+68 K \cos \phi_{0}-96 K \cos ^{3} \phi_{0}}{16 \pi+128 K \cos \phi_{0}}
\end{aligned}
$$

$45^{\circ}$-inclined constriction:

$$
\chi=\frac{2\left|S_{2}^{1} S_{0}^{0}-S_{1}^{0} S_{1}^{1}\right|}{\left(S_{0}^{0}\right)^{2}-\left(S_{1}^{0}\right)^{2}-\left|S_{1}^{1}\right|^{2}}=\frac{8 K \cos ^{2} \phi_{0}\left|\sin \phi_{0}\right|}{\pi+8 K \cos \phi_{0}}
$$

Azimuthal constriction:

$$
\gamma=\frac{\left|S_{2}^{2} S_{0}^{0}-\left(S_{1}^{1}\right)^{2}\right|}{\left(S_{0}^{0}\right)^{2}-\left(S_{1}^{0}\right)^{2}-\left|S_{1}^{1}\right|^{2}}=\frac{4 K \cos ^{3} \phi_{0}}{\pi+8 K \cos \phi_{0}},
$$

Azimuth of maximum fading at $45^{\circ}$ elevation:

$$
\theta_{\phi_{45}}^{\max }=\arg \left\{S_{2}^{1} S_{0}^{0}-S_{1}^{0} S_{1}^{1}\right\}=\theta_{0},
$$

Azimuth of maximum fading at zero elevation:

$$
\theta_{\phi_{0}}^{\max }=\frac{1}{2} \arg \left\{S_{2}^{2} S_{0}^{0}-\left(S_{1}^{1}\right)^{2}\right\}=\theta_{0}+\pi
$$

where $S_{l}^{m}$ is the $l$-th degree, $m$-th order unnormalized complex spherical harmonic coefficient of $p(\theta, \phi)$. The angular spread with the range $[0,1]$ is a measure of how multipath waves concentrate about a single direction; the elevational constriction with the range [-0.5,1] shows how much the multipath APD is concentrated on a single elevational cone or along two paths at same azimuth and opposite elevations; the $45^{\circ}$-inclined constriction with the range $[0,1]$ shows how much the multipath APD is concentrated at the elevational angle $45^{\circ}$; the azimuthal constriction with the range $[0,1]$ shows how much the multipath power is concentrated on two paths on a single elevational cone; the azimuth of maximum fading at $45^{\circ}$ elevation angle and the azimuth of maximum fading at zero elevation angle take values from the intervals $[0, \pi]$ and $[0,2 \pi]$, respectively. 


\section{Spatial Fading Statistics}

\subsection{Fading Rate Variance}

The fading rate variance of a received complex voltage is the simplest statistic that measures the fading rate of a widesense stationary channel, and it can be written as [12]

$$
\begin{aligned}
\sigma_{V}^{2}(\theta, \phi) & =\frac{4 \pi^{2} \Lambda^{2} P_{\mathrm{T}}}{3 \lambda^{2}}\left\{1+\frac{3}{2}\left[\xi\left(2 \sin ^{2} \phi-\frac{2}{3}\right)\right.\right. \\
& +\chi \sin 2 \phi \cos \left(\theta-\theta_{\phi_{4} 5}^{\max }\right) \\
& \left.\left.+\gamma \cos ^{2} \phi \cos 2\left(\theta-\theta_{\phi_{0}}^{\max }\right)\right]\right\}
\end{aligned}
$$

where $\lambda$ is the carrier wavelength. By substituting equations (2)-(7) into (8), the fading rate variance of the 3-D Rician channel can be obtained. For direct comparison with the Rayleigh model, equation (8) can be normalized by the fading rate variance for 3-D omnidirectional APD which can be obtained by setting $K=0$ in (1). According to equations (2)-(5), $\Lambda=1, \xi=1 / 16$, and $\chi=\gamma=0$, if $K=0$. Then the omnidirectional fading rate variance can be written as

$$
\sigma_{V-\mathrm{omni}}^{2}(\theta, \phi)=\frac{\pi^{2} P_{\mathrm{T}}}{4 \lambda^{2}}\left(5+\sin ^{2} \phi\right)
$$

Accordingly, the normalized fading rate variance can be obtained:

$$
\begin{aligned}
\sigma_{N}^{2}(\theta, \phi) & =\frac{\sigma_{V}^{2}(\theta, \phi)}{\sigma_{V-\text { omni }}^{2}(\theta, \phi)} \\
& =\frac{8 \Lambda^{2}}{5+\sin ^{2} \phi}\left[\frac{2}{3}+\xi\left(2 \sin ^{2} \phi-\frac{2}{3}\right)\right. \\
& +\chi \sin 2 \phi \cos \left(\theta-\theta_{\phi_{4} 5}^{\max }\right) \\
& \left.+\gamma \cos ^{2} \phi \cos 2\left(\theta-\theta_{\phi_{0}}^{\max }\right)\right] .
\end{aligned}
$$

\subsection{Level Crossing Rate and Average Fade Du- ration}

The expressions of 2-D spatial LCR and AFD for a Nakagami-m fading signal are reported in [21], and only the fading rate variance $\sigma_{V}(\theta)$ is closely related to the space dimension in the expressions. So, the 3-D LCR and AFD can be obtained by using $\sigma_{V}(\theta, \phi)$ to replace $\sigma_{V}(\theta)$, i.e.

$$
\begin{gathered}
N\left(\rho_{N}, \theta, \phi\right)=\frac{\sigma_{V}(\theta, \phi)}{\sqrt{\pi P_{\mathrm{T}}}} \frac{m^{m-1 / 2}}{\Gamma(m)} \rho_{N}^{2 m-1} \exp \left(-m \rho_{N}^{2}\right) \\
\bar{l}\left(\rho_{N}, \theta, \phi\right)=\frac{\sqrt{\pi P_{\mathrm{T}}} \Gamma\left(m, m \rho_{N}^{2}\right)}{\sigma_{V}(\theta, \phi) m^{m-1 / 2} \rho_{N}^{2 m-1}} \exp \left(m \rho_{N}^{2}\right)
\end{gathered}
$$

where $\rho_{N}=R_{l} / \sqrt{P_{\mathrm{T}}}$ is the normalized threshold level, $R_{l}$ is the envelope threshold, $m$ is the Nakagami-m shape factor, $\Gamma()$ is the Gamma function, and $\Gamma($,$) is the incomplete$ Gamma function. To represent the Rician distribution in terms of the Nakagami-m distribution, we have only to take into consideration the parameter $m$ as this is mentioned inside [22]:

$$
m=\frac{(K+1)^{2}}{2 K+1} \text {. }
$$

Therefore, the approximate LCR and AFD of the 3-D Rician channel can be obtained by substituting (13) into (11) and (12), respectively.

\subsection{Spatial Correlation and Coherence Dis- tance}

According to [23], the spatial correlation function of the 3-D Rician channel at a separation distance $r$ can be written as Mclaurin series

$$
\begin{aligned}
\rho_{c}(r, \theta, \phi) & =1+\frac{\sum_{n=1}^{\infty} \frac{(-1)^{n} r^{2 n}}{(2 n) !} E\left[\left(\frac{d^{n} R}{d r^{n}}\right)^{2}\right]}{E\left(R^{2}\right)-[E(R)]^{2}} \\
& =1-\frac{E\left[\left(\frac{d R}{d r}\right)^{2}\right]}{2\left\{E\left(R^{2}\right)-[E(R)]^{2}\right\}} r^{2}+\cdots \\
& =1-\frac{\sigma_{V}^{2}(\theta, \phi)}{2 \operatorname{Var}_{\text {Ric }}(R)} r^{2}+\cdots
\end{aligned}
$$

where $\operatorname{Var}_{\text {Ric }}(R)$ is the variance of Rician distribution. Meanwhile, following the approaches in [1] and [21], the spatial correlation function can be approximated by an arbitrary Gaussian function and its Mclaurin expansion is

$$
\rho_{c}(r, \theta, \phi) \approx \exp \left[-a\left(\frac{r}{\lambda}\right)^{2}\right] \approx 1-a\left(\frac{r}{\lambda}\right)^{2}+\cdots
$$

where the appropriate constant $a$ is selected such that the terms of (14) and (15) are equal, ensuring that the behavior of both spatial correlation functions are identical for $r$. Accordingly,

$$
\begin{aligned}
\rho_{c}(r, \theta, \phi) & \approx \exp \left[-\frac{\sigma_{V}^{2}(\theta, \phi)}{2 \operatorname{Var}_{\text {Ric }}(R)} r^{2}\right] \\
& =\exp \left\{-\frac{2(K+1) \sigma_{V}^{2}(\theta, \phi)}{P_{\mathrm{T}}\left[4(K+1)-\pi L_{1 / 2}^{2}(-K)\right]} r^{2}\right\}(16)
\end{aligned}
$$

where $L_{1 / 2}(-K)=\exp (-K / 2)\left[(1+K) I_{0}(K / 2)+K I_{1}(K / 2)\right]$ is the Laguerre polynomial, $I_{0}$ is the zeroth order modifed Bessel function of the first kind, and $I_{1}$ is the first order modifed Bessel function of the first kind. 
A measure of the spatial selectivity of wireless channels is its coherence distance at which the spatial correlation drops below a certain level. As in [1] and [12], the coherence distance can be defined as the argument $D_{c}$ at which the approximate correlation (16) drops below the level of $\exp (-1)$ :

$$
D_{c}(\theta, \phi)=\frac{\sqrt{P_{\mathrm{T}}\left[4(K+1)-\pi L_{1 / 2}^{2}(-K)\right]}}{\sigma_{V}(\theta, \phi) \sqrt{2(K+1)}} .
$$

The coherence distance in (17) provides a measure of the needed separation in different 3-D spatial directions for a multiple antenna system employing the spatial diversity.

\section{Results and Discussions}

This section illustrates and analyses the APD, multipath shape factors, fading rate variance of a complex voltage, LCR, AFD, spatial correlation and coherence distance described in Sec. 2 and 3 for 3-D Rician channel through MATLAB simulations. Unless indicated otherwise, the values of the simulation parameters used to obtain the curves are set to $\lambda=0.125 \mathrm{~m}, K=2$ [14], $\theta_{0}=\pi / 4$, and $\phi_{0}=\pi / 6$ [9] for the general urban communication scenarios in the $2.4 \mathrm{GHz}$ frequency band. For ease of analysis, the total power is normalized, i.e., $P_{\mathrm{T}}=1$ in simulations.

\subsection{Multipath Angular Power Density and Mul- tipath Shape Factors}

Figure 2 illustrates the 2-D and 3-D APD of the Rician channel for different directions $(\theta, \phi)$. Figure 3 illustrates the 2-D and 3-D multipath shape factors of the Rician channel for different elevations $\phi_{0}$ of LoS. In the 2-D Rician channel, it is assumed that the AoA of LoS wave is $\theta_{0}$, and the AoA power of NLoS waves is evenly distributed in the azimuth space. Then, the 2-D APD is $p(\theta)=P_{\mathrm{T}}\left[1+2 \pi K \delta\left(\theta-\theta_{0}\right)\right] /[2 \pi(K+1)]$, and 2-D multipath shape factors are the angular spread $\Lambda=\sqrt{2 K+1} /(K+1)$, the angular constriction $\gamma=K /(2 K+1)$ and the azimuthal direction of maximum fading $\theta_{\max }=\theta_{0}$. As mentioned in Sec. 1, typically $90 \%$ of the received power is contained in paths with elevations up to $40^{\circ}$ in urban measurements [9]. However, as shown in Figs. 2 and 3, the 2-D APD and 2-D multipath shape factors can't fully capture the spatial angular direction information, which indicates the disadvantage of the 2-D channel assumption. Figure 3 shows all the curves are symmetric about $\phi_{0}=0$. Besides, the angular spread $\Lambda$, elevational constriction $\xi$ and $45^{\circ}$-inclined constriction $\chi$ have their minimums at $\phi_{0}=0$, and on the contrary, the azimuthal constriction $\gamma$ has a maximum. Equations (1)-(5), Figs. 2 and 3 show that the fading behaviors of the 3-D APD and multipath shape factors mainly depend on the AoA of LoS wave.

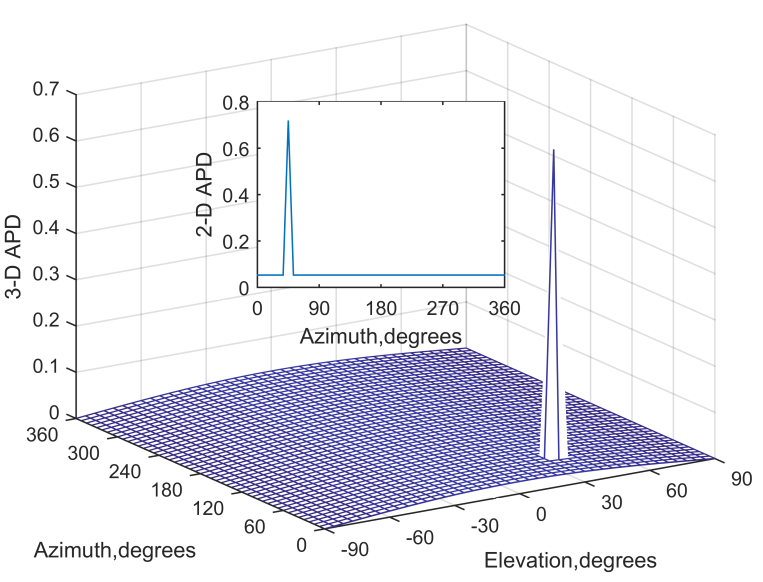

Fig. 2. The multipath angular power density.

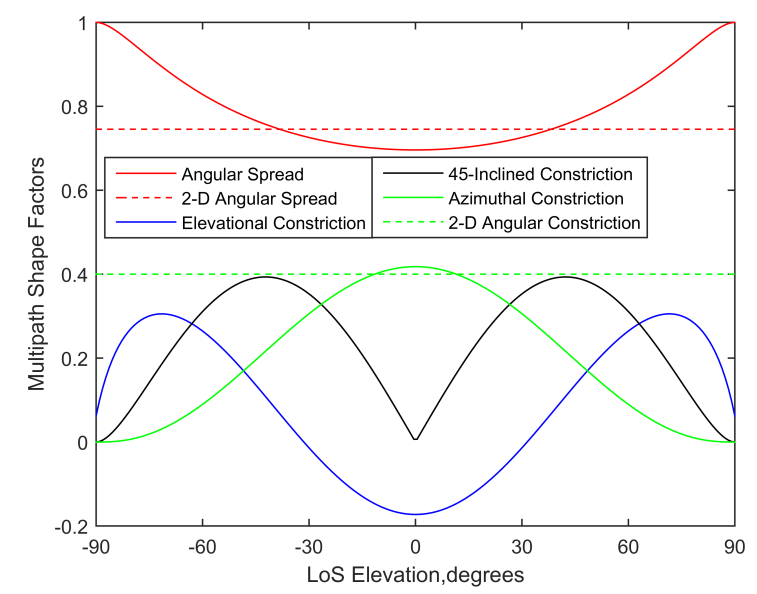

Fig. 3. The multipath shape factors.

\subsection{Fading Rate Variance}

Figure 4 illustrates the root mean square (RMS) normalized fading rate variance in (10) for the different directions $(\theta, \phi)$. Because the elevation angle of $\operatorname{LoS}$ is set to $\theta_{0}=\pi / 4$, the azimuth of maximum fading at $45^{\circ}$ elevation in (6) and the azimuth of maximum fading at zero elevation in (7) are $\theta_{\phi_{45}}^{\max }=\pi / 4$ and $\theta_{\phi_{0}}^{\max }=5 \pi / 4$, respectively. Therefore, as shown in Fig. 4, the RMS normalized fading rate variance has two maximums at $(5 \pi / 4,0)$ and $(\pi / 4, \pi / 4)$ denoting the Rician channel fades fastest here, so the antenna or MIMO beamform pointing should keep away from the two directions to avoid the deep multipath fading in wireless communications. This result would provide useful insight on the design of 3-D MIMO beamforming technology and smart antenna array. In addition, the RMS normalized fading rate variance is a constant at $\phi= \pm \pi / 2$ in the vertical direction, implying a constant average fading rate variance in the horizontal plane. The RMS normalized fading rate variance is less than or equal to 1 , implying that the Rayleigh channel fades faster than the Rician channel in 3-D space. From (2)-(7), Figs. 3 and 4 , we note that the AoA direction of received signal waves has significant effect on the 3-D multipath shape factors and RMS normalized fading rate variance. 


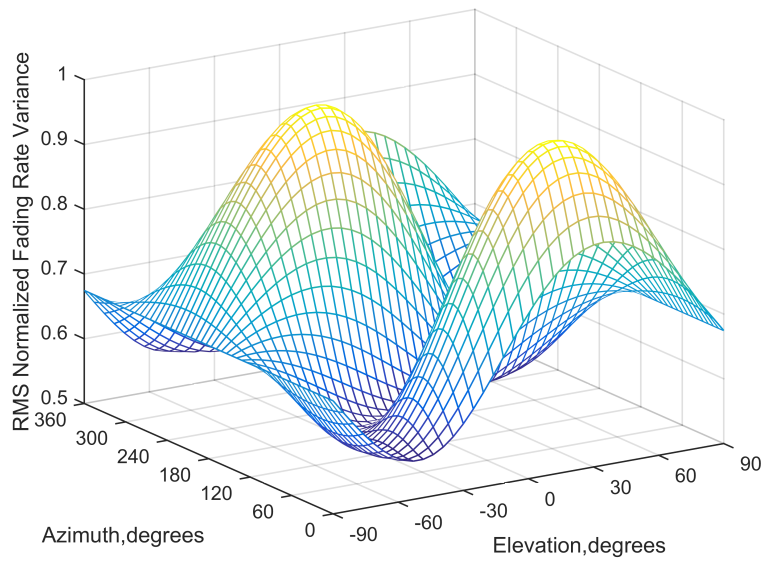

Fig. 4. The RMS normalized fading rate variance.

\subsection{Level Crossing Rate}

Figure 5 illustrates the LCR in (11) for the different directions $(\theta, \phi)$ and normalized threshold levels. The LCR is defined as the average number of the envelope crossing a certain threshold. As shown in Fig. 5 and equation (11), LCR mainly depends on the threshold, and meanwhile it is also very sensitive to the directions $(\theta, \phi)$ at a certain normalized threshold, implying the high dynamic channel fading in 3-D space. Furthermore, Fig. 5 shows that with the increase of normalized threshold, LCR increases directly at a certain elevation angle and different azimuth angles, but increases in fluctuation at a certain azimuth angle and different elevation angles, implying the dynamic behaviors of Rician channel are different in the horizontal plane and the vertical plane. As derived in (12), AFD is inversely proportional to the LCR, so its dynamic behavior can be inferred from Fig. 5 .

\subsection{Spatial Correlation and Coherence Dis- tance}

Figure 6 illustrates the spatial correlation in (16) for the different directions $(\theta, \phi)$ and normalized separation distances. As shown in Fig. 6, the maximum spatial correlations are achieved at $r<0.1 \lambda$ where the spatial correlations almost have nothing to do with directions $(\theta, \phi)$. The spatial correlation decreases as the normalized distance increases away from $0.1 \lambda$, and furthermore, the spatial correlation decreases faster in the horizontal plane than the vertical plane in 3-D space, implying the spatial correlation in the vertical plane is larger than that in the horizontal plane for the same separation distance. The spatial correlation is also sensitive to the directions $(\theta, \phi)$, and this is valuable to 3-D MIMO beamforming technology. Moreover, Fig. 6 shows that $0.5 \lambda$ is the proper spacing between two adjacent antenna elements of the antenna array. Figure 7 illustrates the coherence distance in (17) with the spatial correlation coefficient $\exp (-1)$ for the different directions $(\theta, \phi)$. The coherence distance provides a measure of the needed separation in a multiple antenna system employing the spatial diversity.

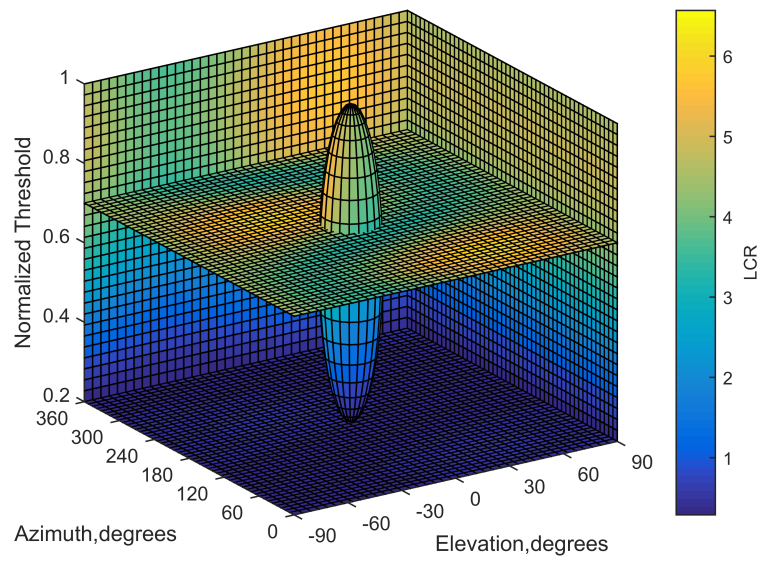

Fig. 5. The level crossing rate.

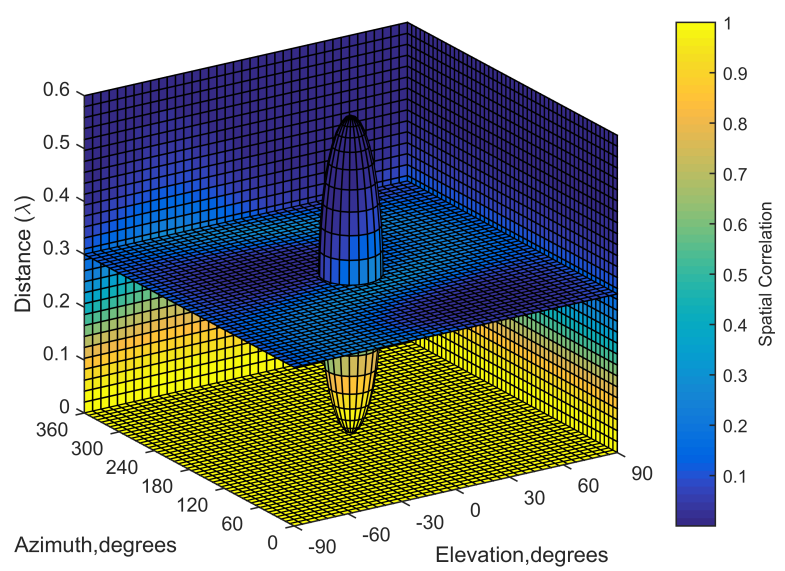

Fig. 6. The spatial correlation.

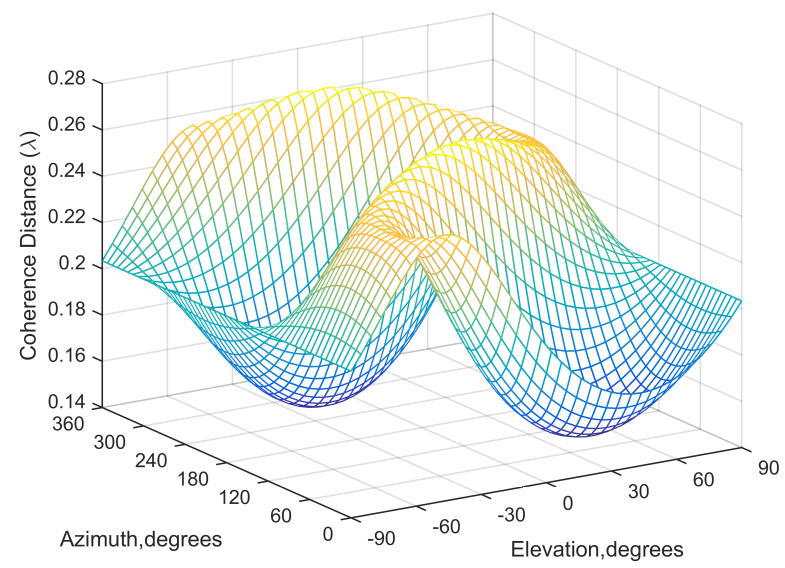

Fig. 7. The coherence distance.

As shown in Fig. 7, the coherence distance is a constant at $\phi= \pm \pi / 2$ in the vertical direction, implying a constant coherence distance in the horizontal plane, but it varies greatly in the other directions. The explicit angular dependence of the coherence distance suggests that different separations may be needed in different 3-D spatial directions for the antenna array design. 


\section{Conclusion}

A 3-D APD model comprising a LoS component and a NLoS component has been proposed to analyze the spatial selectivity of Rician channel. Based on the APD model, 3-D multipath shape factors have been derived and greatly simplified the descriptions of the fading statistics like the fading rate variance, level crossing rate, average fade duration, spatial correlation and coherence distance. The simulation analysis shows that the 3-D spatial angular directions have significant effect on these fading statistics of Rician channel. The results would provide useful insight on the design of 3-D MIMO beamforming technology and smart antenna array for the future $5 \mathrm{G}$ cellular networks.

\section{Acknowledgments}

This work was funded by the National Natural Science Foundation of China No. 91438104, No. 61501065, No. 61571069 and No. 61601067, the Fundamental Research Funds for the Central Universities No. 106112016CDJX160 001 and the Chongqing Research Program of Basic Research and Frontier Technology No. CSTC2016JCYJA0021.

\section{References}

[1] DURGIN, G. D., RAPPAPORT, T. S. Theory of multipath shape factors for small-scale fading wireless channels. IEEE Transactions on Antennas and Propagation, 2000, vol. 48, no. 5, p. 682-693. DOI: $10.1109 / 8.855486$

[2] LU, J., HAN, Y. Application of multipath shape factors in Nakagami$\mathrm{m}$ fading channel. In Proceedings of the IEEE International Conference on Wireless Communications and Signal Processing. Nanjing (China), 2009, p. 1-4. DOI: 10.1109/WCSP.2009.5371524

[3] SHANG, H., HAN, Y., LU, J. Statistical analysis of Rician and Nakagami-m fading channel using multipath Shape factors. In Proceedings of the IEEE International Conference on Computational Intelligence and Natural Computing Proceedings (CINC). Wuhan (China), 2010, p. 398-401. DOI: 10.1109/CINC.2010.5643809

[4] MORAITIS, N., CONSTANTINOU, P., VOUYIOUKAS, D. Power angle profile measurements and capacity evaluation of a SIMO system at $60 \mathrm{GHz}$. In Proceedings of the 21st Annual IEEE International Symposium on Personal, Indoor and Mobile Radio Communications. Instanbul (Turkey), 2010, p. 1027-1031. DOI: 10.1109/PIMRC.2010.5672088

[5] MORAITIS, N., VOUYIOUKAS, D., CONSTANTINOU, P. Indoor angular profile measurements and channel characterization at the millimeter-wave band. In Proceedings of the IEEE 5th European Conference on Antennas and Propagation (EUCAP). Rome (Italy), 2011, p. 155-159. ISBN: 978-88-8202-074-3

[6] LONI, Z. M., ULLAH, R., KHAN, N. M. Analysis of fading statistics based on angle of arrival measurements. In Proceedings of the IEEE 2011 International Workshop on Antenna Technology (iWAT). Hong Kong (China), 2011, p. 314-319. DOI: 10.1109/IWAT.2011.5752364

[7] LONI, Z. M., KHAN, N. M. Analysis of fading statistics in cellular mobile communication systems. The Journal of Supercomputing, 2013, vol. 64, no. 2, p. 295-309. DOI: 10.1007/s11227-012-0799-1
[8] RODRIGUEZ, I., ALMEIDA, E. P. L., ABREU, R., et al. Analysis and comparison of $24 \mathrm{GHz}$ cmWave radio propagation in urban and suburban scenarios. In Proceedings of the IEEE Wireless Communications and Networking Conference (WCNC). Doha (Qatar), 2016, p. 1-7. DOI: $10.1109 /$ WCNC.2016.7564893

[9] FUHL, J., ROSSI, J. P., BONEK, E. High-resolution 3-D direction of arrival determination for urban mobile radio. IEEE Transactions on Antennas and Propagation, 1997, vol. 45, no. 4, p. 672-682. DOI: $10.1109 / 8.564093$

[10] FLEURY, B. H. First- and second-order characterization of direction dispersion and space selectivity in the radio channel. IEEE Transactions on Information Theory, 2000, vol. 46, no. 6, p. 2027-2044. DOI: $10.1109 / 18.868476$

[11] PAL, A., BEACH, M., NIX, A. A novel quantification of 3D directional spread from small-scale fading analysis. In Proceedings of the IEEE International Conference on Communications. Istanbul (Turkey), 2006, p. 1699-1704. DOI: 10.1109/ICC.2006.254964

[12] VACHLEV, D. G., BRADY, D. Three-dimensional multipath shape factors for spatial modeling of wireless channels. IEEE Transactions on Wireless Communications, 2009, vol. 8, no. 11, p. 5542-5551. DOI: 10.1109/TWC.2009.080988

[13] GULFAM, S. M., NAWAZ, S. J., AHMED, A., et al. Analysis on multipath shape factors of air-to-ground radio communication channels. In Proceedings of the IEEE Wireless Telecommunications Symposium (WTS). London (UK), 2016, p. 1-5. DOI: $10.1109 /$ WTS.2016.7482050

[14] ZHOU, T., TAO, C., LIU, L., et al. Ricean k-factor measurements and analysis for wideband high-speed railway channels at $2.35 \mathrm{GHz}$. Radioengineering, 2014, vol.23, no. 2, p. 578-585. DOI: 10.13164/re

[15] RAIDA, Z., KOLKA, Z., MARSALEK, R., et al. Communication subsystems for emerging wireless technologies. Radioengineering, 2012, vol. 21, no. 4, p. 1036-1049. DOI: 10.13164/re

[16] CHRONOPOULOS, S. K., CHRISTOFILAKIS, V., TATSIS, G., et al. Performance of turbo coded OFDM under the presence of various noise types. Wireless Personal Communications, 2016, vol. 87, no. 4, p. 1319-1336. DOI: 10.1007/s11277-015-3055-1

[17] CHRONOPOULOS, S. K., TATSIS, G., KOSTARKIS, P. Turbo coded OFDM with large number of subcarriers. Journal of Signal and Information Processing, 2012, vol. 3, no. 2, p. 161-168. DOI: $10.4236 /$ jsip.2012.32021

[18] WANG, W., LIANG, D., WANG, Z., et al. Design and implementation of a FPGA and DSP based MIMO radar imaging system. Radioengineering, 2015, vol. 24, no. 2, p. 518-526. DOI: $10.13164 /$ re.2015.0518

[19] IVANIS, P., DRAJIC, D., BRKIC, S. Cross-layer combining of adaptive modulation and truncated ARQ in multichannel beamforming MIMO systems. Radioengineering, 2015, vol. 24, no. 4, p. 1050-1059. DOI: $10.13164 /$ re.2015.1050

[20] CLARKE, R. H., KHOO, W. L. 3-D mobile radio channel statistics. IEEE Transactions on Vehicular Technology, 1997, vol. 46, no. 3, p. 798-799. DOI: 10.1109/25.618205

[21] CHEN, Y., MUCCHI, L., WANG, R., et al. Modeling network interference in the angular domain: Interference azimuth spectrum. IEEE Transactions on Communications, 2014, vol. 62, no. 6, p. 2107-2120. DOI: 10.1109/TCOMM.2014.2314651

[22] NAKAGAMI, M. The m-distribution-A general formula of intensity distribution of rapid fading. Statistical Method of Radio Propagation, 1960, p. 3-36. DOI: 10.1016/B978-0-08-009306-2.50005-4

[23] PAPOULIS, A., PILlAI, S. U. Probability, Random Variables and Stochastic Processes. 1st ed., New York (USA): MiGraw-Hill, 2002. ISBN: 0071122567 
About the Authors ...

Derong DU received the B.E. degree in automation from the Chengdu University, Chengdu, China, in 2010 and the M.S. degree in control theory and control engineering from Guilin University of Electronic Technology, Guilin, China, in 2013. $\mathrm{He}$ is currently working towards the Ph.D. degree with the College of Communication Engineering, Chongqing University, Chongqing, China. His research interests include mobile radio channel modeling, multiple-input-multiple-output systems and fifth generation $(5 \mathrm{G})$ cellular networks.

Xiaoping ZENG (corresponding author) received the B.E., M.S., and Ph.D. degrees in Electrical Engineering from Chongqing University, Chongqing, China in 1982, 1987, and 1996, respectively. He is now a professor and Ph.D. supervisor at the College of Communication Engineering, Chongqing University, China. His research interests include aeronautical information network, multiple-input-multipleoutput systems and space information network.
Xin JIAN received his B.E. and Ph.D. degrees from Chongqing University, Chongqing, China in 2009 and 2014, respectively. $\mathrm{He}$ is now an associate professor at the College of Communication Engineering, Chongqing University, China. His interests include antenna and propagation, narrow band internet of things and the next generation mobile communication.

Feng YU received the B.E. degree in communication engineering from Wuhan University, Wuhan, China, in 2016. She is currently working towards the M.S. degree with the College of Communication Engineering, Chongqing University, Chongqing, China. Her research interests include mobile radio channel modeling and the next generation mobile communication.

Lijuan MIAO received the B.E. degree in electronic information engineering, Chongqing University, Chongqing, China, in 2015. She is currently working towards the M.S. degree with the College of Communication Engineering, Chongqing University, Chongqing, China. Her research interests include multiple-input-multiple-output systems, aeronautical information network and space information network. 